\title{
Avaliação do nível de ruído em instalações para suínos ${ }^{1}$
}

\author{
Carlos A. de P. Sampaion ${ }^{2}$ Irenilza de A. Nääs ${ }^{3}$, Douglas D. Salgado ${ }^{4}$ \& Marcos P. G. Queirós ${ }^{5}$
}

\begin{abstract}
RESUMO
Esta pesquisa foi realizada em instalações para suínos nas fases de creche e terminação, com o objetivo de se avaliar e comparar o nível de ruído emitido pelos animais principalmente nos horários de arraçoamento e ruídos provenientes de outras fontes, estimando-se os riscos de exposição ocupacional. A avaliação se deu com auxílio de um medidor de nível de pressão sonora posicionado na altura de 1,50 m, considerada altura média do ouvido humano, conforme preceitua a NR-15. Avaliaram-se quatro instalações, sendo duas para creche e duas para terminação de suínos. A análise estatística foi feita pelo modelo fatorial e teste de médias. Os resultados indicaram diferença significativa a $1 \%$ de probabilidade entre as instalações. O ruído médio encontrado foi de $65 \mathrm{~dB}(\mathrm{~A})$ e de $70 \mathrm{~dB}(\mathrm{~A})$ nas instalações para creche e terminação, respectivamente, que se enquadrou dentro do limite permitido pela NR-15, que é de $85 \mathrm{db}(\mathrm{A})$ para uma jornada de trabalho de $8 \mathrm{~h}$ diárias. Considerando-se o ruído de pico observado nos horários de alimentação e vacinação e, principalmente na fase de terminação, indica-se a necessidade de uso de equipamento de proteção auricular. Notou-se tendência do ruído ser mais intenso em condições de desconforto térmico.
\end{abstract}

Palavras-chave: fatores ambientais, produção animal, salubridade, suíno

\section{Evaluation of the noise level in swine housing}

\begin{abstract}
This research was carried out with in growing and finishing facilities of swine housing with the objective to evaluate and compare the noise level emitted by the animals mainly during feeding and noise level from other sources, and estimate the occupational risk exposition of workers. The evaluation was made using noise level equipment and measurement as indicated in NR-15 norm located at $1.50 \mathrm{~m}$ of height, corresponding to the mean height of the worker's ear. Four swine housings were evaluated. The statistical analysis was done using fatorial design and mean test. The results indicated significant differences at $1 \%$ probability level among the four housings. The mean noise level was $65 \mathrm{~dB}(\mathrm{~A})$ and $70 \mathrm{~dB}(\mathrm{~A})$ for growing and finished housing respectively, within the limits allowed in the NR15 norm (85 dB(A)) for a daily working of $8 \mathrm{~h}$. Considering the peak noise level observed during feeding and other task schedule mainly finishing swine housing ear protection is recommended. There was also a tendency for the noise level to increase as thermal conditions became more unconfortable.
\end{abstract}

Key words: environmental factors, animal production, healthy, swine

\footnotetext{
1 Trabalho de pesquisa financiado pela FAPESP, CNPq e CAPES.

2 CAV/UDESC. Av. Luiz de Camões, 2090, Bairro Conta Dinheiro, CEP 88520-000, Lages, SC. Fone: (49) 2101-9100. E-mail: a2caps@cav.udesc.br

${ }^{3}$ FEAGRI/UNICAMP. Cidade Universitária Zeferino Vaz, Bairro Barão Geraldo, CEP 13083-970 Campinas, SP. Fone: (19) 3521-2900. E-mail: irenilza@agr.unicamp.br

${ }^{4}$ Estatístico. Pós-Graduando da FEAGRI/UNICAMP. Fone: (19) 3521-2900. E-mail: overdoug@yahoo.com.br

${ }^{5}$ Bolsista PIBIC. Graduando da FEAGRI/UNICAMP. Fone: (19) 3521-2900. E-mail: mpgq@agr.unicamp.br
} 


\section{INTRODUÇÃO}

A partir da década de setenta, a produção de suínos no Brasil começou a ser realizada em sistema de confinamento, objetivando-se melhorar o controle sanitário, reduzir a perda energética dos animais e aumentar a produtividade. Como conseqüência da relação animal confinado versus ambiente, desconhecida para as condições brasileiras, eliminaram-se as opções de busca por parte dos animais de um ambiente mais propício ao seu bem-estar, desde então, vários estudos foram realizados no intuito de se conhecer a interação animal-ambiente-instalação e otimizar o sistema produtivo.

Os trabalhadores da agroindústria, mais particularmente das edificações para animais, de maneira geral estão expostos a riscos para a sua integridade física e psicológica e isto implica em seu afastamento da empresa por períodos de tempo consideráveis, prejudicando a si próprio e gerando prejuízos também para as empresas. Informações sobre ruídos e seus efeitos no bem-estar do animal e do trabalhador em sistemas de produção intensiva de animais são escassas e uma visão do ambiente de trabalho facilita a compreensão das dificuldades, desconfortos, insatisfações e a ocorrência de acidentes e doenças ocupacionais (Marchant et al., 2001).

No Brasil, o Ministério de Trabalho e Emprego, baseado em estudos específicos, normatiza índices que possibilitam executar o trabalho com maior conforto e segurança, propiciando satisfação, saúde e eficiência ao trabalhador, com penalidades às empresas que não se adequarem às normas (Brasil, 1998). Yaremchuk et al. (1997) descrevem que o nível médio de ruído pode não indicar riscos para a audição humana e sugerem a medição dos ruídos de impacto e de pico para indicar esses riscos.

Fisicamente, os sons desagradáveis e indesejáveis podem ser definidos como ruído, pois causam incômodos e neuroses, afetam física e psicologicamente o indivíduo e dependendo dos níveis causam ao trabalhador, lesões auditivas irreversíveis podendo levá-lo a uma surdez permanente (Gerges, 1992; PMAC, 1994).

Tendo em vista os prejuízos que o ruído pode causar às pessoas que lhe estão expostas, faz-se necessário tomar medidas para reduzir ao máximo a intensidade da pressão sonora nos ambientes de trabalho, sendo que a maneira mais freqüente de se solucionar o problema é o fornecimento de protetores auriculares para os trabalhadores (Vieira, 1997), embora o método mais eficaz e correto seja atuar no ambiente de trabalho, reduzindo o nível de ruído na fonte como forma preventiva. Segundo a norma brasileira (NR15), para ruídos de até $85 \mathrm{~dB}(\mathrm{~A})$, o trabalhador pode se expor durante toda a jornada de trabalho diária (8 horas); acima deste nível, começam a surgir riscos para os trabalhadores além de ruídos intensos; acima de $90 \mathrm{~dB}(\mathrm{~A})$, dificultam a comunicação verbal, aumentando a tensão psicológica (Iida, 1990).

De acordo com Gustafsson (1997), ruído acima de $100 \mathrm{~dB}(\mathrm{~A})$ tem sido verificado na alimentação de suínos de forma manual. Os sons emitidos pelos animais indicam também uma situação indesejável, como que emitindo sofrimento, fome, doença, medo, desconforto, estado emocional e, con- seqüentemente, os gritos podem indicar distúrbio no seu bemestar (Weary et al., 1999).

Objetivou-se com esta pesquisa, avaliar e comparar, estatisticamente, o nível de ruído na produção de suínos e estimar os riscos da exposição ocupacional, em instalações de creche e terminação.

\section{MATERIAL E MÉTODOS}

Este trabalho foi desenvolvido em duas granjas de produção de suínos comercial, denominadas G1 e G2, localizadas no município de Salto, interior de São Paulo, correspondendo a quatro instalações: duas para a fase de creche e duas para a fase de terminação, nas condições de verão e inverno de 2003. O município de Salto encontra-se na latitude de $23^{\circ} 12^{\prime} 10^{\prime}$ ' Sul e longitude $47^{\circ} 17^{\prime} 35^{\prime}$ ' Oeste, com altitude média de $521 \mathrm{~m}$. O clima predominante da região de acordo com Köppen é Cwa, quente, temperado chuvoso, com estação seca no inverno e verão quente.

A G1 possuía um padrão de arraçoamento manual na creche e na terminação nos seguintes horários: às 7 h 15min, 9 h, 10 h 30 min, 12 h 30 min e 15 h, cuja ração era colocada no cocho em quantidade predeterminada e umedecida em seguida sendo, no último trato, oferecida às 16 h $30 \mathrm{~min}$ na forma seca. A G2 possuía um padrão de arraçoamento manual na creche realizado às 7 h 15 min, 9 h, 10 h 30 min, 12 e 15 h, em que a ração era posta no cocho em quantidade predeterminada e umedecida logo após e, oferecida no último trato, às $16 \mathrm{~h}$ na forma seca e, na terminação, às 7 h 15 min, 10 h e 12 h 30 min, cuja ração se punha no cocho em quantidade também predeterminada como as anteriores e umedecida para, em seguida, ser oferecida no último trato, às 16 h, na forma seca.

\section{Características das instalações para suínos na fase de creche}

Na G1, a instalação tinha orientação leste-oeste, paredes de alvenaria, pé-direito no beiral de 2,30 m, telhado de duas águas com lanternim e cobertura de telhas de fibrocimento, beiral de $0,85 \mathrm{~m}$, piso de concreto e corredor externo. A instalação era formada de salas com área de piso de $173,00 \mathrm{~m}^{2}$, fechamento lateral de alvenaria na altura de $1,00 \mathrm{~m}$ e cortina para controle ambiental, cuja limpeza era feita diariamente, pela manhã, com raspagem e, a lavação do piso era feita na entrada de um novo lote de animais. A sala alojava 360 suínos de genética formada por fêmea (Landrace $\mathrm{x}$ Large White) e macho ( Pic $^{\circledR}$ ), perfazendo $0,48 \mathrm{~m}^{2}$ por animal; no verão, o peso médio dos suínos era de $15,20 \mathrm{~kg}$ perfazendo $31,62 \mathrm{~kg} \mathrm{~m}^{-2}$ piso e, no inverno, seu peso médio era de $17,70 \mathrm{~kg}$, totalizando $38,39 \mathrm{~kg} \mathrm{~m}^{-2}$ piso.

Na G2, a instalação tinha orientação leste-oeste, paredes de alvenaria, pé-direito no beiral de 2,35 m, telhado de duas águas com lanternim e cobertura de telhas de barro, beiral de 0,35 m, piso cimentado e corredor central. A instalação era formada de salas com área de piso de $61,25 \mathrm{~m}^{2}$, dividida em catorze baias de piso semi-ripado, fechamentos laterais de alvenaria na altura de 1,55 m e cortina para controle ambiental, sendo a limpeza feita diariamente, pela manhã, com raspagem e lavação. A 
sala alojava 140 leitões de genética formada por fêmea (Camborough) e macho (AgPic) perfazendo $0,36 \mathrm{~m}^{2}$ por animal, sendo que no verão o peso médio dos suínos era de $22,73 \mathrm{~kg}$, perfazendo $51,95 \mathrm{~kg} \mathrm{~m}^{-2}$ piso e, no inverno, seu peso médio era de 20,26 kg, totalizando $46,31 \mathrm{~kg} \mathrm{~m}^{-2}$ piso.

\section{Características das instalações para suínos na fase de terminação}

Na G1, a instalação utilizada possuía orientação leste-oeste, pé-direito no beiral de 2,00 m, telhado de duas águas com cobertura de telhas de fibrocimento, correspondendo a 2/3 da área do telhado, beiral de $0,10 \mathrm{~m}$, corredor de serviço elevado e piso cimentado. A instalação era formada de baias com área de piso de 324,00 $\mathrm{m}^{2}$, separadas por tábuas de madeira, com limpeza diária pela manhã, além de raspagem e lavação do piso realizada uma vez por semana. A instalação, que alojava uma média de 200 animais por baia, perfazendo 1,62 $\mathrm{m}^{2}$ por animal, no verão alojava 1.600 animais com peso médio de $75 \mathrm{~kg}$, perfazendo 46,29 $\mathrm{kg} \mathrm{m}^{-2}$ piso e, no inverno, 950 animais com peso médio de $70 \mathrm{~kg}$, totalizando $43,21 \mathrm{~kg} \mathrm{~m}^{-2}$ piso.

Na G2, a instalação utilizada possuía orientação leste-oeste, pé-direito no beiral de 3,20 m, telhado de duas águas com cobertura de telhas de fibrocimento, beiral de $0,25 \mathrm{~m}$, corredor central e piso cimentado e era formada de baias com área de piso de $24,20 \mathrm{~m}^{2}$ separadas por paredes na altura de $1,10 \mathrm{~m}$ e cortina para controle ambiental. No fundo das baias havia uma depressão que formava uma lâmina de água e a limpeza das baias consistia no esgotamento da lâmina de água. A instalação, com uma média de 25 animais por baia, perfazendo $0,97 \mathrm{~m}^{-2}$ por animal, alojava no verão 1.175 animais com peso médio de $70 \mathrm{~kg}$, perfazendo $58,49 \mathrm{~kg} \mathrm{~m}^{-2}$ piso e, no inverno, recebia 950 animais com peso médio de $70 \mathrm{~kg}$, totalizando $47,28 \mathrm{~kg} \mathrm{~m}^{-2}$ piso.

\section{Medidas, análise e modelo estatístico}

Os dados de conforto térmico (Monitor de Estresse Térmico 34 Quest) no interior da instalação foram avaliados pelo Índice de Bulbo Úmido e Temperatura de Globo - IBUTG (Minard et al., 1971), a temperatura e umidade relativa do ar externo (higrotermo-anemômetro HTA 4.200 Pacer), coletados a cada 30 minutos, no período total.

As avaliações do nível de ruído emitido pelos animais (medidor de nível de pressão sonora Quest 2900) foram feitas com sensor posicionado na altura $1,50 \mathrm{~m}$ do piso, no centro da edificação, conforme as especificações da NR-15 do Ministério do Trabalho e Emprego (1978), cujos dados foram coletados em intervalos de 5 min em decibéis $(\mathrm{dB}(\mathrm{A}))$, durante toda a jornada de trabalho. O ruído de impacto foi medido em decibéis linear (dB-L) e o ruído de fundo analisado conforme descreve Gerges (1992) com as edificações vazias.

Realizou-se a comparação dos ambientes de creche e terminação para o nível de ruído pelo modelo fatorial $2 \times 4$ × 6 , representando as estações (E.: verão, inverno), galpões (G: G1c, G2c, G1t e G2t, correspondentes às edificações de creche e terminação das granjas G1 e G2), horários (H: 7 h 8 h, 8 h 30 min - 9 h 30 min, 9 h 30 min - 10 h 30 min, 11 h 30 min - 12 h 30 min, 14 h 30 min - 15 h 30 min e 16 - 17 h) e três dias de repetições, conforme modelo estatístico:

$$
\begin{gathered}
Y_{\mathrm{ij} \ell}=\mu+\alpha+\alpha_{\mathrm{i}}(\mathrm{E})+\beta_{\mathrm{j}}(\mathrm{G})+\sigma_{\ell}(\mathrm{H})+\alpha \beta_{\mathrm{ij}}\left(\mathrm{E}^{*} \mathrm{G}\right)+\alpha \sigma_{\mathrm{i} \ell}\left(\mathrm{E}^{*} \mathrm{H}\right)+ \\
\beta \sigma_{\mathrm{j} \ell}\left(\mathrm{G}^{*} \mathrm{H}\right)+\alpha \beta \sigma_{\mathrm{ij} \ell}\left(\mathrm{E}^{*} \mathrm{G}^{*} \mathrm{H}\right)+\xi_{\mathrm{ijk} \ell}
\end{gathered}
$$

em que: $\mathrm{Y}_{\mathrm{ij} \ell}$ - variável resposta (nível de ruído); $\mu$ - média geral de $\mathrm{Y}_{\mathrm{ij} \ell} ; \alpha_{\mathrm{i}}$ - efeito do nível i do fator estação; $\beta_{\mathrm{j}}$ - efeito do nível j do fator galpão; $\sigma_{l}$ - efeito do nível $\ell$ do fator horário; $\alpha \beta_{\mathrm{ij}} ; \alpha \sigma_{\mathrm{il}} ; \sigma_{\mathrm{j} \ell} ; \alpha \beta \sigma_{\mathrm{ij} \ell}$ - efeito das interações entre os níveis; $\xi_{\mathrm{ij} \ell}$ - erro independente das variáveis aleatórias com distribuição normal $\left(0 ; \sigma^{2}\right)$

As formas de condução dos processos (manejo) nas instalações, que são repetitivos e seguem um padrão, não sofreram qualquer interferência, mas serviram de base para as comparações e inferências estatísticas. As análises foram realizadas através do programa estatístico SAS.

\section{RESULTADOS E DISCUSSÃO}

O nível de ruído de fundo encontrado no interior das instalações vazias é mostrado na Figura 1. Verifica-se que a

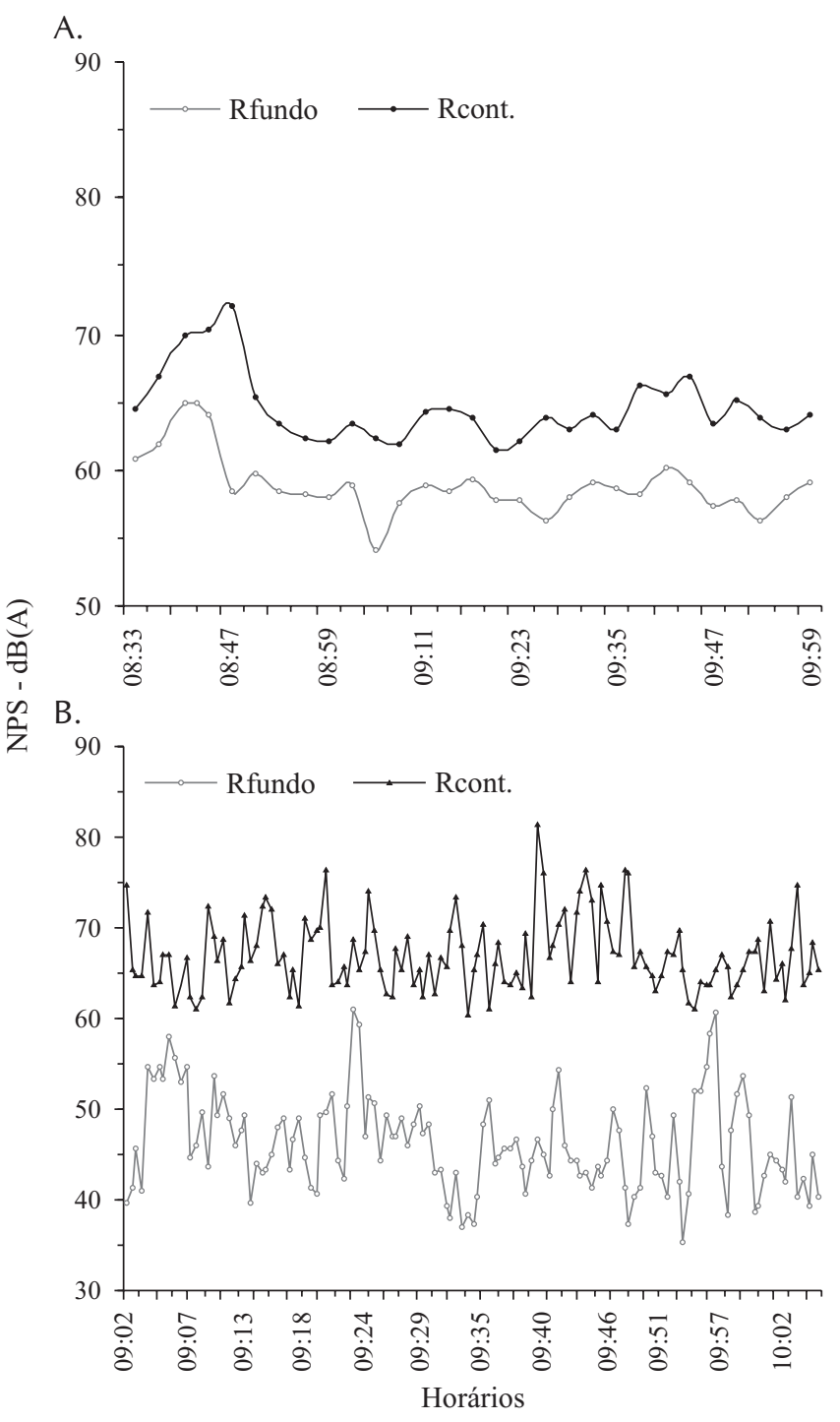

Figura 1. Ruído de fundo versus ruído contínuo na creche $(A)$ e na terminação (B) 
diferença entre os ruídos médios e de fundo estão na faixa de $10 \mathrm{~dB}(\mathrm{~A})$, não havendo ruído de outras fontes que possam mascarar o ruído de interesse, que é o emitido pelos animais, conforme descreve Gerges (1992).

As Figuras 2A e B mostram a evolução dos ruídos de impacto e de pico nas instalações de creche, para as condições de verão e inverno, respectivamente.

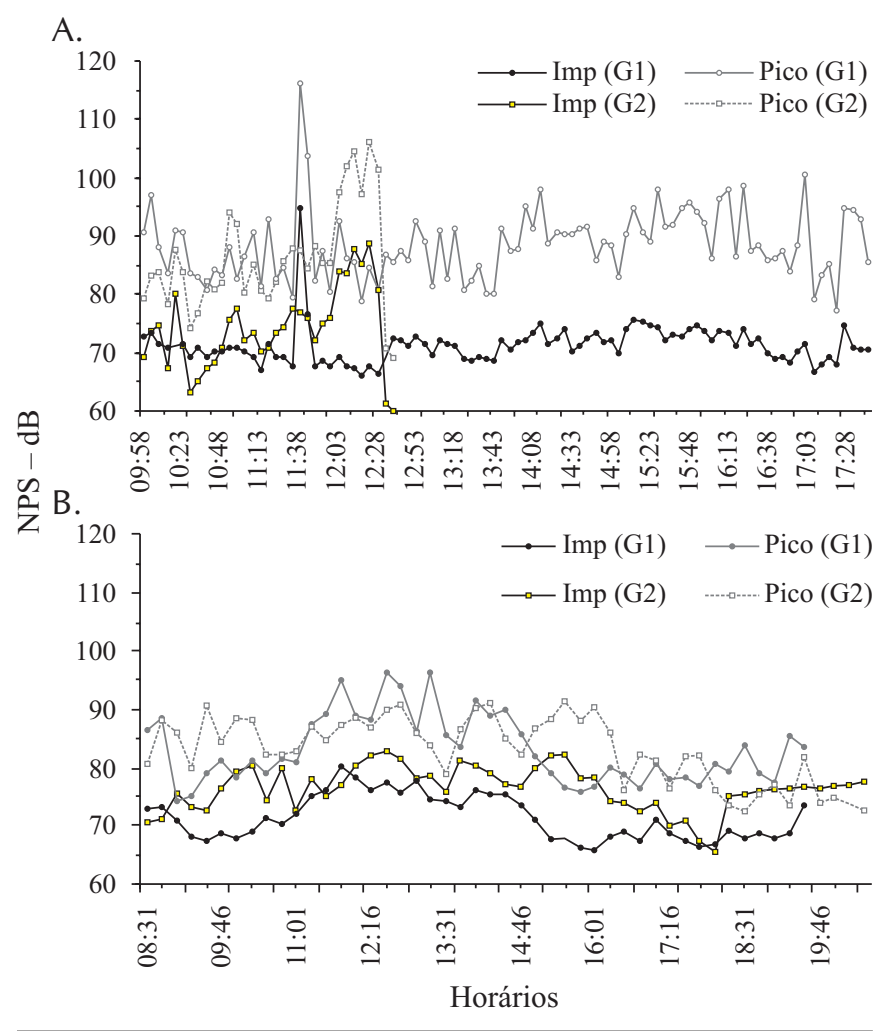

Figura 2. Ruído de impacto e de pico em creche de suínos nas condições de verão $(A)$ e inverno $(B)$

Nota-se que o ruído de impacto ficou abaixo do limite estabelecido pela NR-15/78 do Ministério do Trabalho e Emprego, que é de $130 \mathrm{~dB}$ (linear) e o ruído de pico também não ultrapassou o mesmo limite, constatando-se significativa oscilação durante o dia e tendência de serem mais elevados no verão e, ainda, de acompanhar as condições de desconforto térmico nas instalações.

As Figuras 3A e B mostram a evolução dos ruídos de impacto e de pico nas instalações de terminação, para as condições de verão e inverno, respectivamente.

Como observado na creche, o ruído de impacto ficou abaixo do limite estabelecido pela NR-15 do Ministério do Trabalho e Emprego (1978) e o ruído de pico também não ultrapassou o mesmo limite. Os valores desses ruídos oscilaram significativamente durante o dia, com tendência de estarem mais elevados no verão e de acompanhar as condições de desconforto térmico nas instalações, conforme encontraram Weary et al. (1999), que apontam a vocalização excessiva em suínos como sendo indicativo de uma situação de medo ou estresse.

A análise das Figuras 2 e 3, mostra que os níveis de ruído de pico, que estão acima de $85 \mathrm{~dB}$ e até superior a $100 \mathrm{~dB}$,
A.

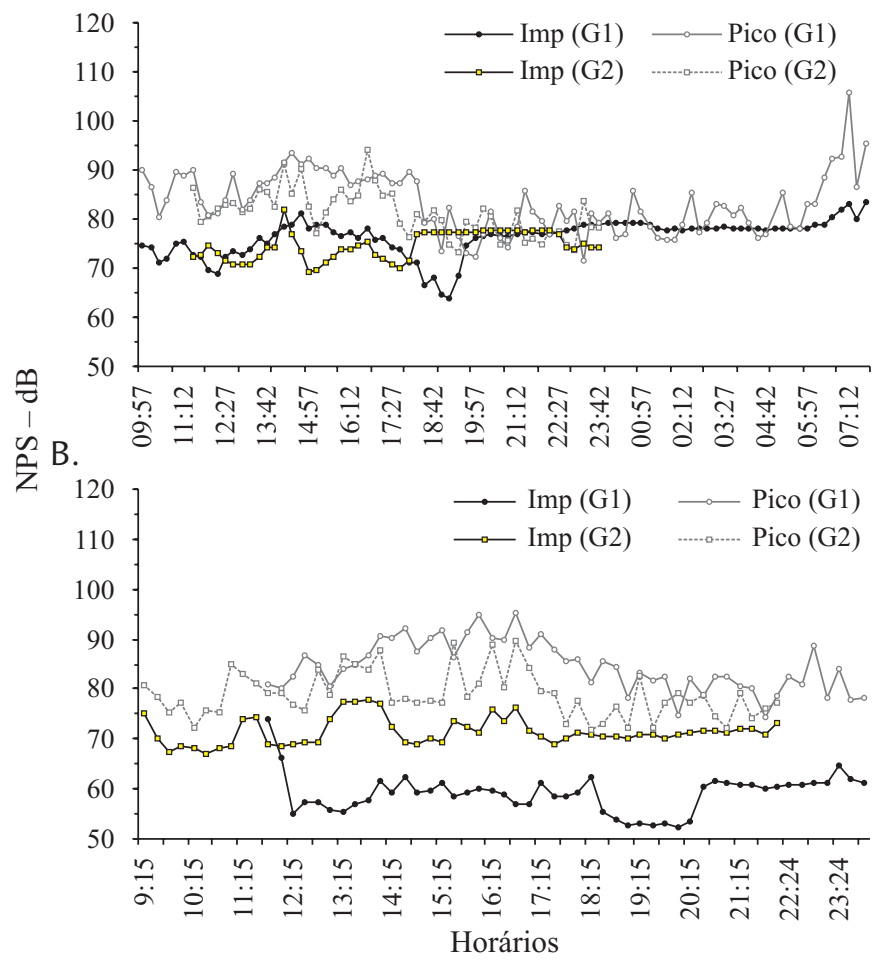

Figura 3. Ruído de impacto e de pico em terminação de suínos nas condições de verão (A) e inverno (B)

chamam para uma atenção e prevenção dos trabalhadores envolvidos nessas atividades, muito embora as normas não façam referências específicas a esses ruídos. Para a situação de exposição dos animais, Gustafsson (1997) indica que pressões sonoras acima de $100 \mathrm{~dB}(\mathrm{~A})$ encontradas nos horários de arraçoamento indicam a necessidade de lutar por comida, o que é um indicativo negativo na questão de bem-estar animal.

Tanto nas edificações para creche como para terminação, percebem-se comportamentos diferentes dos ruídos de impacto e de pico e isto pode estar relacionado principalmente às características construtivas das instalações, ou seja, o ambiente exerce influência na pressão acústica devido à introdução de absorção e reflexões.

Os resultados da análise de variância para Índice de bulbo úmido e temperatura de globo (IBUTG) e ruído médio (Rcont.) são apresentados na Tabela 1.

Verifica-se que a estação do ano (E), instalação (G) e os

Tabela 1. Resultados das análises de variância para a média horária de IBUTG e Ruído contínuo ( $\mathrm{R}_{\text {cont. }}$ )

\begin{tabular}{cccc}
\hline \multirow{2}{*}{ F.V. } & G.L. & \multicolumn{2}{c}{ Quadrados Médios } \\
\cline { 3 - 4 } E & 1 & $1108,69^{* *}$ & $\mathbf{R}_{\text {cont. }}$ \\
G & 3 & $117,20 * *$ & $195,75^{* *}$ \\
H & 5 & $159,71^{* *}$ & $190,25^{* *}$ \\
Ex G & 3 & $51,48^{* *}$ & $71,50^{* *}$ \\
Ex H & 5 & $4,04^{*}$ & 7,13 \\
GxH & 15 & $14,06^{* *}$ & $51,24 * *$ \\
Ex GxH & 15 & $2,81^{*}$ & $29,94 * *$ \\
\hline
\end{tabular}

* significativo a $5 \%$ de probabilidade; ** significativo a $1 \%$ de probabilidade 
Tabela 2. Resultados do teste de comparação de médias para o nível de ruído no ambiente das instalações das granjas e as condições de temperaturas e umidades médias do ar interno

\begin{tabular}{|c|c|c|c|c|c|c|c|}
\hline \multirow{2}{*}{ Altura } & & \multicolumn{6}{|c|}{ Horários/Ruído, dB } \\
\hline & & $7: 00-8: 00$ & $8: 30-9: 30$ & $9: 30-10: 30$ & $11: 30-12: 30$ & $14: 30-15: 30$ & $16: 00-17: 00$ \\
\hline \multicolumn{8}{|c|}{ Edificação: Creche } \\
\hline \multirow{2}{*}{1,5 (G1) } & Ver. & $66,7 a b c$ & $66,3 \mathrm{bcd}$ & $64,5 \mathrm{bcd}$ & $61,0 \mathrm{~d}$ & $68,8 a b$ & $67,0 a b c$ \\
\hline & Inv. & $67,4 a b c$ & $68,6 a b c$ & $66,1 \mathrm{bcd}$ & $62,2 \mathrm{~d}$ & $68,0 a b c$ & $67,1 a b c$ \\
\hline \multirow{2}{*}{1,5 (G2) } & Ver. & $63,1 \mathrm{bcd}$ & $63,6 \mathrm{bcd}$ & $63,7 \mathrm{bcd}$ & $65,0 \mathrm{bcd}$ & $68,2 a b c$ & $72,2 \mathrm{a}$ \\
\hline & Inv. & $65,7 \mathrm{bc}$ & $61,7 d$ & $63,7 \mathrm{bcd}$ & $63,0 \mathrm{~cd}$ & $67,3 a b c$ & $65,0 \mathrm{bcd}$ \\
\hline \multicolumn{8}{|c|}{ Edificação: Terminação } \\
\hline \multirow{2}{*}{1,5 (G1) } & Ver. & $73,3 a b$ & $70,7 \mathrm{bc}$ & 67,5 cde & $64,5 \mathrm{def}$ & $70,4 b c$ & $70,3 b c$ \\
\hline & Inv. & $69,2 \mathrm{bcd}$ & $68,9 \mathrm{bcd}$ & $68,4 \mathrm{bcd}$ & $62,6 \mathrm{ef}$ & 66,2 cdef & 66,0 cdef \\
\hline \multirow{2}{*}{1,5 (G2) } & Ver. & $70,4 b c$ & $77,9 \mathrm{a}$ & $70,8 b c$ & 67,4 cde & 68,0 bcde & $69,1 \mathrm{bcd}$ \\
\hline & Inv. & $68,6 \mathrm{bcd}$ & $70,4 b c$ & $61,3 f$ & $62,6 \mathrm{ef}$ & $68,6 \mathrm{bcd}$ & $68,9 \mathrm{bcd}$ \\
\hline \multirow{2}{*}{$\left.\mathrm{T}_{\mathrm{m}} .{ }^{\circ} \mathrm{C}\right) / \mathrm{UR}_{\mathrm{m} .} .(\%)$} & & $23,0 / 92,0$ (v) & $23,5 / 90,5(\mathrm{v})$ & $24,5 / 88,0$ (v) & $25,0 / 81,5$ (v) & $27,0 / 72,5$ (v) & $26,5 / 75,5$ (v) \\
\hline & & 19,5/ 80,0 (i) & $19,0 / 74,0$ (i) & $22,5 / 60,0$ (i) & 23,8/57,0 (i) & $26,0 / 46,5$ (i) & $25,3 / 52,5$ (i) \\
\hline
\end{tabular}

- Letras iguais nas linhas e nas colunas não diferem pelo teste de Tukey a nível de $5 \%$ de probabilidade

horários (H) exerceram influência significativa sobre os valores obtidos para ruído e, como a interação tripla foi significativa, evidencia uma dependência entre as variáveis e, neste caso, foi necessário um desdobramento da interação para avaliar o seu comportamento, mostrado na Tabela 2.

Foram poucas as medidas que diferiram significativamente com relação ao nível de ruído contínuo entre os horários e as estações. Verificou-se que os menores valores de ruídos acontecerem nos horários intermediários e, os maiores nos horários extremos, embora com pequenas diferenças, fato relacionado, possivelmente, aos aspectos de manejo da criação, que possuem várias características semelhantes nas granjas G1 e G2. Como observado para o ruído de impacto, o ruído contínuo se manteve nos limites recomendados pelas normas NR-15 do Ministério do Trabalho e Emprego (1978) podendo-se considerar o ambiente salubre. Verificou-se tendência de ocorrer ruídos mais intensos nas horas de maior calor interno nas instalações.

Nas edificações para terminação verificaram-se ruídos pouco mais elevados na granja G2 e isto pode estar relacionado sobretudo às características construtivas da instalação, ocasionando maior nível de pressão sonora (NPS) em virtude da existência de paredes, ou seja, o ambiente exerce influência na pressão acústica pela introdução de absorção e reflexões. Da mesma maneira que o encontrado para ruído de impacto, o ruído contínuo se manteve nos limites recomendados pelas normas NR-15/78, podendo-se considerar o ambiente salubre.

\section{CONCLUSÕES}

1. O ruído médio se manteve nos limites recomendados pela norma NR-15, que é de $85 \mathrm{~dB}(\mathrm{~A})$, podendo-se considerar o ambiente salubre, o mesmo ocorrendo com o ruído de impacto.

2. O ruído de pico observado durante a alimentação e em algumas etapas de produção, sobretudo nas instalações de terminação, indica a necessidade de uso de protetor auricular.

3. Foi notória a tendência do ruído emitido pelos suínos acompanhar as condições de desconforto térmico nas instalações.

\section{LITERATURA CITADA}

Brasil. Consolidação das leis do trabalho. 23.ed. São Paulo: Saraiva, 1998. 193p.

Gerges, S. N. Y. Ruído: Fundamentos e controle. Florianópolis: DEM, UFSC. 1992. 576p.

Gustafsson, B. The health and safety of workers in a confined animal system. Livestock Production Science, Amsterdan, v.49, p.191-202, 1997.

Iida, I. Ergonomia: Projeto e produção. São Paulo: Edgard Blucher, 1990. 465p.

Marchant, J. N.; Whittakerb, X; Broom, D. M. Vocalisations of the adult female domestic pig during a standard human approach test and their relationships with behavioural and heart rate measures. Applied Animal Behaviour Science, Londres, v.72, n.2, p.23-39, 2001.

Minard, D.; Goldsmith R.; Farrier, P. H. Jr., Lambiotte, BJ, Jr. Physiological evaluation of industrial heat stress. American Industrial Hygiene Association Journal, v.32, p.17-28, 1971.

Ministério do Trabalho e Emprego. Portaria 3.214 de Jul. 1978. Normas regulamentadoras de segurança e saúde no trabalho - NR-15: Atividades e operações insalubres. Brasília, 1978. http://www.mte.gov.br/temas/segsau/legislacao. 10 Nov. 2001.

PMAC, Exposição ao ruído: Norma para a proteção de trabalhadores que trabalham em atividades com barulho. Revista Proteção, Rio de Janeiro, v.6, n.29, p.136-138, 1994.

Vieira, S. D. G. Análise ergonômica do trabalho em uma empresa de fabricação de móveis tubulares. Estudo de caso. Florianópolis: UFSC, 1997. Dissertação Mestrado

Weary, D. M.; Appleby, M. C.; Fraser, D. Responses of piglets to early separation from the sow. Applied Animal Behaviour Science, Londres, v.63, n.4, p.289-300, 1999.

Yaremchuk, K.; Dickson, L.; Burk, K. Noise level analysis of commercially available toys. Journal of Pediatric Otorhinolaryngology, Toronto, v.41, p.187-197, 1997. 